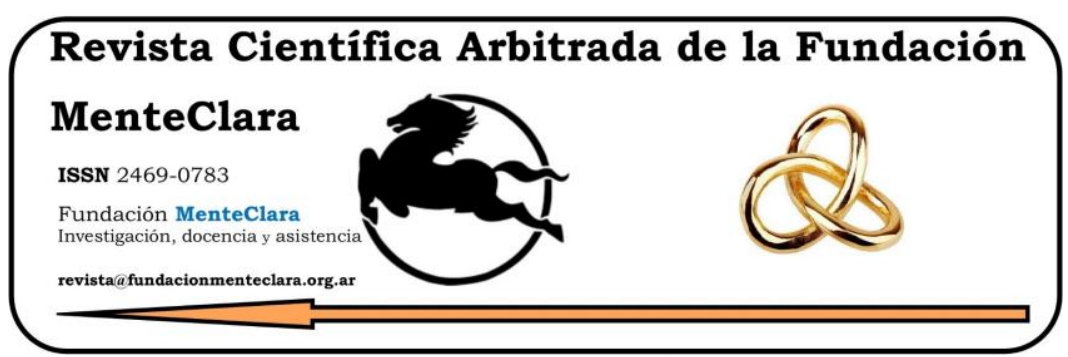

Artículos atravesados por (o cuestionando) la idea del sujeto -y su género- como una construcción psicobiológica de la cultura. Articles driven by (or questioning) the idea of the subject -and their gender-as a cultural psychobiological construction.

Vol. 6 (2021), enero-diciembre

ISSN 2469-0783

https: / / datahub.io/dataset/2021-6-e241

\title{
BIENESTAR SUBJETIVO EN EL ENVEJECIMIENTO ACTIVO
}

\author{
SUBJECTIVE WELL-BEING IN ACTIVE AGING \\ Mara González Bosque mara.gonzalez@uflouniversidad.edu.ar \\ Estudiante de Psicología, Cátedra Psicología de la Salud, Universidad de Flores, \\ Argentina.
}

Cómo citar este artículo / Citation: González Bosque, M. (2021). Bienestar subjetivo en el Envejecimiento Activo. Revista Científica Arbitrada de la Fundación MenteClara, Vol. 6 (241). DOI: https://doi.org/10.32351/rca.v6.241

Copyright: (C) 2021 RCAFMC. Este artículo de acceso abierto es distribuido bajo los términos de la licencia Creative Commons Attribution 4.0 International License (CC BY 4.0). Recibido: 23/07/2021. Aceptado: 24/07/2021 Publicación online: 25/07/2021

\section{Resumen}

Estudios mostraron que el bienestar está ligado a factores diversos como condiciones de crianza, vínculos con parientes y amigos, tipo de actividades laborales, rasgos de personalidad, disponibilidad y utilización del tiempo libre, lugar donde se vive, acceso a parques, lugares de convivencia social, los niveles de seguridad, la tenencia de hijos y sus diferentes edades, los vínculos de pareja, la economía del hogar y personal, el marco macroeconómico, la distribución de los ingresos, el desempleo y la ocupación, el nivel de salud, la escala de valores que se posee, la disponibilidad de participación en tomas de decisiones a nivel político y más. El bienestar subjetivo afecta y marca fuertemente el grupo etario de 60 a 64 años, relacionado con los cambios propios de la etapa evolutiva en la que se introducen, el bienestar subjetivo en dicha etapa aparece equilibrado y tendiente al incremento y el nivel educativo de la persona mayor tiene una directa relación con el bienestar subjetivo experimentado, porque a medida que éste se incrementa tienen mayor posibilidad de alcanzar el bienestar. Evaluando 30 años de estudios, la definición de envejecimiento activo y con éxito, se siguen sosteniendo. Más allá de las diferencias en las definiciones y miradas encontradas en la literatura, hay que destacar un punto de encuentro en relación a la necesidad de superar las restricciones de los modelos basados en visiones determinadas por lo 
cultural y social, delimitándose a los lugares donde se han evaluado, y no universalmente, también destacar la intención de integrar criterios objetivos con la subjetividad de aquellos implicados, con modelos más inclusivos que den la oportunidad de envejecer dignamente a todas las personas por igual. Resaltando la necesidad de una solidaridad intergeneracional y aproximación a esta cuestión desde un proceso de enseñanza y aprendizaje durante el transcurso de toda la vida.

\section{Abstract}

Studies have shown that well-being is linked to diverse factors such as conditions of upbringing, ties with relatives and friends, type of work activities, personality traits, availability and use of free time, place where one lives, access to parks, places of social coexistence, levels of security, having children and their different ages, couple ties, household and personal economy, macroeconomic framework, income distribution, unemployment and occupation, level of health, scale of values held, availability of participation in decision making at the political level, and more. The subjective well-being strongly affects and marks the age group from 60 to 64 years, related to the changes of the evolutionary stage in which they are introduced, the subjective well-being in that stage appears balanced and tends to increase and the educational level of the older person has a direct relationship with the subjective wellbeing experienced, because as this increases they have a greater possibility of achieving well-being. Evaluating 30 years of studies, the definition of active and successful aging still holds. Beyond the differences in the definitions and views found in the literature, it is necessary to highlight a meeting point in relation to the need to overcome the restrictions of models based on visions determined by the cultural and social, delimited to the places where they have been evaluated, and not universally, also highlight the intention to integrate objective criteria with the subjectivity of those involved, with more inclusive models that give the opportunity to age with dignity to all people equally. Highlighting the need for intergenerational solidarity and approaching this issue from a lifelong teaching and learning process.

Palabras Claves: bienestar subjetivo; envejecimiento activo; adultos mayores; transcurso de la vida; cambios biopsicosociales

Keyw ords: subjective well-being; active aging; older adults; course of life; biopsychosocial changes 


\section{Introducción}

En el siguiente artículo de revisión bibliográfica, se elaboró una articulación entre dos variables, Bienestar Subjetivo, que se define como la autoevaluación que hace la persona sobre su vida, dicha valoración está constituida por juicios cognitivos sobre satisfacción vital. Así como valoraciones afectivas de los estados de ánimo y emociones propias. En tanto podemos decir que en el bienestar subjetivo se hallan relacionados los componentes afectivo y cognitivo Diener et al (2011), y Envejecimiento Activo, que lo podemos definir como el mantenimiento de la capacidad funcional, la práctica de actividades físicas, el control o la ausencia de enfermedades crónicas, la aceptación de los cambios biopsicosociales, la salud, la autonomía, la independencia y la creatividad, evitar la depresión, estar contento con el entorno familiar y tener amigos, la inserción social y la búsqueda de medios de aprendizaje. Colussi et al., (2019). La Organización Mundial de la Salud entiende por "Envejecimiento saludable es un proceso continuo de optimización de oportunidades para mantener y mejorar la salud física y mental, la independencia y la calidad de vida a lo largo de la vida". (OPS/OMS, 2021).

\section{Bienestar Subjetivo}

Se define al bienestar Subjetivo como la autoevaluación que hace la persona sobre su vida, dicha valoración está constituida por juicios cognitivos sobre satisfacción vital. Así como valoraciones afectivas de los estados de ánimo y emociones propias. En tanto podemos decir que en el bienestar subjetivo se hallan relacionados los componentes afectivo y cognitivo, en el componente afectivo, las personas con bienestar subjetivo, declaran la experimentación frecuente de emociones positivas 
y pocas vivencias de emociones negativas. En relación al componente cognitivo, dirian que están satisfechas con su vida Diener et al (2011). Según algunos autores existen dos importantes acercamientos a la conceptualización del bienestar la hedónicay eudaimónica, la primera se basa en sentirse bien y la segunda en funcionar bien, ambas se complementan por lo que el abordaje completo del bienestar implica que ambas sean tenidas en cuenta. (Ryan \& Deci, E. , 2001).

Otros autores agregan que, en la perspectiva basada en sentirse bien, la hedónica, el acercamiento empírico más relevante es el llamado bienestar subjetivo, porque destaca que la percepción subjetiva que las personas tienen de su vida es más importante, que los indicadores objetivos, sobre la calidad de vida. Las personas que se autodenominan felices y así se sienten, son vistas por otras personas como personas felices, recuerdan muchos más momentos felices, ríen más y tienen respuestas fisiológicas relacionadas con el sentimiento de felicidad Lyubomirsky et al (2005).

En la visión de la siguiente autora, aparecen propuestas seis dimensiones de las cuales surgen y se desarrollan las escalas de bienestar psicológico (Ryff, 1989). Estas son: la autonomía-capacidad de mantener la independencia y la individualidad en diferentes ámbitos/situaciones y sobrellevar la presión social-; dominio del ambiente -capacidad de seleccionar o crear ambientes que favorezcan la satisfacción de metas y necesidades-; autoaceptación -tener un registro real de las propias acciones, motivos y sentimientos-; relaciones positivas con los otros -confianza mutua y empatía-; crecimiento personal -ocupación del desarrollo del potencial personal para seguir creciendo-; y propósito de vida -proponerse metas y marcar objetivos para direccionar la vida- (Keyes et al, 2002) 
Estudios mostraron el bienestar está ligado a factores diversos como condiciones de crianza, vínculos con parientes y amigos tipo de actividades laborales rasgos de personalidad, disponibilidad y utilización del tiempo libre, lugar donde se vive, acceso a parques, lugares de convivencia social, los niveles de seguridad, la tenencia de hijos y sus diferentes edades, los vínculos de pareja, la economía del hogar y personal, el marco macroeconómico, la distribución de los ingresos, el desempleo y la ocupación, el nivel de salud, la escala de valores que se posee, la disponibilidad de participación en tomas de decisiones a nivel político y más. (Rojas, 2011).

\section{Envejecimiento Activo}

Podemos decir que el Envejecimiento Activo es el arte de vivir bien con una buena calidad de vida. Entre los principales factores de este proceso de envejecimiento están el mantenimiento de la capacidad funcional, la práctica de actividades físicas, el control o la ausencia de enfermedades crónicas, la aceptación de los cambios biopsicosociales, la salud, la autonomia, la independencia y la creatividad, evitar la depresión, estar contento con el entorno familiar y tener amigos, la inserción social y la búsqueda de medios de aprendizaje. Colussi et al (2019).

Según otros autores podemos agregar que el conjunto de los acontecimientos vitales, y el cómo han vivido, determinan el cómo las personas llegan a su vejez. Es por esto que no se considera el envejecimiento cómo un estado, sino como un proceso dinámico activo que se deriva de la forma particular de cada persona de envejecer y atribuir significado a este proceso, lo que genera que se vivencie de diferentes formas. (Limon Mendizabal, 2018). 
La visión de este constructo inicialmente se planteó como una adaptación a actividades y actitudes, de las personas mayores, como alternativa a los roles que fueron perdiendo debido a la edad, para poder mantener un sentido optimista y positivo de sí mismos. (Havighurst, 1963).

Evaluando 30 años de estudios, la definición de envejecimiento activo y con éxito, se siguen sosteniendo. Más allá de las diferencias en las definiciones y miradas encontradas en la literatura, hay que destacar un punto de encuentro en relación a la necesidad de superar las restricciones de los modelos basados en visiones determinadas por lo cultural y social, delimitado a los lugares donde se han evaluado, y no universalmente, también destacar la intención de integrar criterios objetivos con la subjetividad de aquellos implicados con modelos más inclusivos que den la oportunidad de envejecer dignamente a todas las personas por igual. Petretto et al (2016).

\section{Bienestar subjetivo AND Envejecimiento Activo}

Los habitantes del planeta de más de 60 años, se cuadruplicarian entre los años 1950 al 2050. Para entonces se pronostica un in crescendo del proceso de envejecimiento. (Organización Panamericana de la Salud, 2021). Por esto algunos autores agregan que más allá del activismo y participación que poseen consecuencias positivas en el envejecimiento, las investigaciones de ocio y envejecimiento plantean también la necesidad de tomar en cuenta no sólo el envejecimiento activo sino también satisfactorio. La participación en actividades significativas y con sentido para el individuo. Monteagudo et al (2014).

Aquí agregaremos que según investigaciones son numerosos los beneficios que aporta la actividad física en los adultos mayores en lo físico 
y en lo psicológico, haciendo hincapié en lo relacionado con el bienestar subjetivo y la depresión en las personas adultas. Guillen Pereira et al (2018).

Otros autores coinciden en la necesidad de una solidaridad intergeneracional y aproximación a esta cuestión desde un proceso de enseñanza y aprendizaje durante el transcurso de la vida. Un envejecimiento activo y satisfactorio con una valiosa experiencia de óseo, encuentra un apoyo favorable en un recorrido vital que se moldea en la infancia, atraviesa la adolescencia y se consolida en la juventud y madurez para iniciar un envejecimiento en plenitud (Cohen \& Stebbins 2013).

El bienestar subjetivo afectay marca fuertemente el grupo etario de 60 a 64 años, relacionado con los cambios propios de la etapa evolutiva en la que se introducen, el bienestar subjetivo en dicha etapa aparece equilibrado y tendiente al incremento y el nivel educativo de la persona mayor tiene una directa relación con el bienestar subjetivo experimentado, porque a medida que éste se incrementa tienen mayor posibilidad de alcanzar el bienestar Hung et al (2021).

\section{Conclusión}

Para concluir el presente trabajo y teniendo en cuenta que los habitantes del planeta de más de 60 años, se cuadruplicarán entre los años 1950 al 2050., junto con el hecho de que el conjunto de los acontecimientos vitales, y el cómo han vivido, determinan el cómo las personas llegan a su vejez. Es importante considerar la interdependencia de las dos variables analizadas, ya que poseen una estrecha relación, tanto el Envejecimiento Activo, así como el Bienestar Subjetivo, necesitan el uno del otro para alcanzarse de la manera más óptima. Resaltando la 
necesidad de una solidaridad intergeneracional y aproximación a esta cuestión desde un proceso de enseñanza y aprendizaje durante el transcurso de toda la vida, más allá del activismo y participación que poseen consecuencias positivas en el envejecimiento, las investigaciones de ocio y envejecimiento plantean también la necesidad de tomar en cuenta no sólo el envejecimiento activo sino también satisfactorio junto con la participación en actividades significativas y con sentido para el individuo.

Conflicto de intereses: Ninguno que declarar. 


\section{Referencias}

Colussi, E., Pichler, N. A., \& Grochot, L. (2019). Perceptions of elderly and their relatives about aging. Revista Brasileira de Geriatria e Gerontologia, 1. Obtenido de https:/ / www.scielo.br/scielo.php?script=sci_serial\&pid=1809-9823\&lng=en\&nrm=iso

Diener, E., Lucas, R. E., \& Oishi, S. (2011). Subjective well-being: The science of happiness and life satisfaction. OUP USA: Second Edition.

Guillén Pereira, L., Bueno Fernández, E., Gutiérrez Cruz, M., \& Guerra Santiesteban, J. (2018). Programa de actividad fisica y su incidencia en la de presión y bienestar subje tivo de adultos mayores. Federación Española de Asociaciones de Docentes de Educación Física, 14-19. Obtenido de https://dialnet.unirioja.es/servlet/articulo?codigo=6367717

Havighurst, R. J. (1963). Successful aging. Gerontologist, 8-13.

Hung, G. J., Alvarez, Y. T., \& Cedeño, D. C. (2021). Bienestar subjetivo en la persona mayor. Una intervención psicosocial. Revista científica Olimpia, 436-450. Obtenido de https://revistas.udg.co.cu/index.php/olimpia/article/view/2304

Limon Mendizabal, M. R. (2018). Envejecimiento activo: un cambio de paradigma sobre el envejecimiento y la vejez. Aula Abierta, 45-54. Obtenido de https://dialnet.unirioja.es/servlet/articulo?codigo $=6292831$

Lyubomirsky, S., King, L, \& Diener, E. (2005). The benefi ts of frequent positive affect: Does happiness lead to success? Psychological Bulletin, 803-855. doi:http://dx.doi. org/10.1037/0033-2909.131.6.803

Monteagudo, M. J., Cuenca Amigo, J., \& San Salvador del Valle, R. (2014). Aportaciones del ocio al envejecimiento satisfactorio. 1-191.

OPS/OMS. (2021). Envejecimiento y ciclo de vida. Organizacion mindial de la salud. Obtenido de https://www.who.int/ageing/about/facts/es/

Organización Panamericana de la Salud. (2021). Envejecimiento Saludable. Organización mundial de la Salud. Obtenido de https://www.paho.org/es/temas/envejecimiento-saludable

Pereira, L. G., Fernandez, E. B., Cruz, M. G., \& Santiesteban, J. R. G. (2018). Programa de actividad física y su incidencia en la de presión y bienestar subjetivo de adultos mayores. Retos: nuevas tendencias en educación física, deporte y recreación, 14-19. Obtenido de https://dialnet.unirioja.es/servlet/articulo?codigo $=6367717$

Petretto, D. R., Pili, R., Gaviano, L., Matos Lopez, C., \& Zuddas, C. (2016). Envejecimiento activo y de éxito o saludable : una breve historia de modelos conceptuales. Revista Española de Geriatría y Gerontología, 229-241. Obtenido de https://www.sciencedirect.com/science/article/abs/pii/S0211139X1500205X

Rojas, M. (2011). El bienestar subje tivo: su contribución a la apreciación y la consecución del progreso y el bienestar humano. Realidad, datos y espacio. Revista internacional de estadistica y geografia, 64-77. Obtenido de https://rde.inegi.org.mx/rde_02/doctos/rde_02_art4.pdf

Ryan, R., \& Deci, E. . (2001). On happiness and human potentials: A review of research on hedonic and eudaimonic well-being. Annual Review of Psychology, 141-166. doi:http://dx.doi. org/10.1146/annurev.psych.52.1.141

Ryff, C. D. (1989). Happiness is everything, or is it? Explorations on the meaning of psychological well-being. Journal of Personality and Social Psychology, 1069-1081. 